\title{
AFTERWORD: DYNAMICAL ZETA FUNCTIONS FOR AXIOM A FLOWS
}

\author{
SEMYON DYATLOV AND COLIN GUILLARMOU
}

Abstract. This afterword addresses the previously published article "Differentiable dynamical systems" by S. Smale, which appeared in the Bulletin of the American Mathematical Society, 73 (1967), no. 6, 747-817.

We show that the Ruelle zeta function of any smooth Axiom A flow with orientable stable/unstable spaces has a meromorphic continuation to the entire complex plane. The proof uses the meromorphic continuation result of DyGu16 together with CoEa71,GMT17, which imply that every basic hyperbolic set can be put into the framework of DyGu16].

\section{Preliminaries}

Let $\mathcal{M}$ be a compact manifold, and let $X$ be a $C^{\infty}$ vector field on $\mathcal{M}$. Denote by $\varphi^{t}=\exp (t X)$ the corresponding flow. In this note we prove

Theorem. Assume that $\varphi^{t}$ is an Axiom A flow (see Definition 2.3) with orientable stable/unstable spaces. Define the Ruelle zeta function

$$
\zeta(\lambda)=\prod_{\gamma^{\sharp}}\left(1-e^{-\lambda T_{\gamma^{\sharp}}}\right), \quad \Re \lambda \gg 1,
$$

where the product is taken over the primitive closed orbits $\gamma^{\sharp}$ of $\varphi^{t}$, with the exception of fixed points, and $T_{\gamma^{\sharp}}$ are the corresponding periods. Then $\zeta(\lambda)$ extends meromorphically to $\lambda \in \mathbb{C}$.

We refer the reader to $\$ 2$ for examples of Axiom A flows and for the dynamical systems terminology used here.

The above theorem was first conjectured by Smale [Sm67, bottom of p. 802]. It was proved in the special case of Anosov flows by Giulietti, Liverani, and Pollicott GLP13. Dyatlov and Zworski DyZw16 gave a simple microlocal proof in the Anosov case. In our previous work DyGu16, we generalized the microlocal techniques used in DyZw16 to handle the case of open hyperbolic systems, defined as locally maximal hyperbolic sets with a smooth neighborhood on which the flow is strictly convex. We emphasize that the introduction of microlocal tools and the use of escape functions for the study of hyperbolic diffeomorphisms and flows appeared first in the works of Faure, Roy, and Sjöstrand FRS08 and Faure and Sjöstrand

Received by the editors March 19, 2018.

2010 Mathematics Subject Classification. Primary 37D20.

This research of the first author was conducted during the period he served as a Clay Research Fellow.

The second author was partially supported by the ANR project ANR-13-JS01-0006 and by the ERC consolidator grant IPFLOW. 
FaSj11; they were inspired by the works of Kitaev [Ki99, Blank, Keller, and Liverani BKL02, Liverani Li04, Butterley and Liverani [BuLi07, Gouëzel and Liverani GoLi08, and Baladi and Tsujii BaTs07] that used anisotropic Sobolev/Hölder spaces adapted to the dynamics. The meromorphic extension of the zeta function for a particular case of Axiom A flow, namely the Grassmannian extension of a contact Anosov flow, was also proved by Faure and Tsujii FaTs17.

See the introduction to DyGu16 for an overview of results on dynamical zeta functions and Pollicott-Ruelle resonances, and see the book by Baladi Ba17] for the related case of hyperbolic maps.

The proof in this note combines DyGu16 with the results of Conley and Easton CoEa71 and Guillarmou, Mazzucchelli, and Tzou [GMT17] which show that every locally maximal hyperbolic set has a smooth neighborhood with a strictly convex flow, as well as with Smale's spectral decomposition for Axiom A flows.

We note that just like in DyGu16, one can consider a more general Ruelle zeta function with a potential. In fact, the methods of DyGu16 apply to even more general dynamical traces and twisted zeta functions associated to the action of $X$ on vector bundles; see DyGu16, §5.1, Theorem 4]. Moreover, the assumption that $X$ is $C^{\infty}$ can be relaxed to $C^{k}$ for large $k=k\left(C_{0}\right)$ if we only want to continue the Ruelle zeta function to a half-plane $\left\{\Re \lambda \geq-C_{0}\right\}$. Finally, the orientability hypothesis holds in many natural cases (such as geodesic flows on orientable negatively curved manifolds), and it can be removed under certain topological assumptions by using a twisted zeta function; see [GLP13, Appendix B].

\section{REVIEW OF HYPERBOLIC DYNAMICS}

In this section we briefly review several standard definitions and facts from the theory of hyperbolic dynamical systems. We refer the reader to KaHa97 for a comprehensive treatment of hyperbolic dynamics.

Assume that $\mathcal{M}$ is a compact $C^{\infty}$ manifold without boundary and $\varphi^{t}=\exp (t X)$ is a $C^{\infty}$ flow generated by a vector field $X$.

Definition 2.1. Let $\mathcal{K} \subset \mathcal{M}$ be a compact $\varphi^{t}$-invariant set. We say that $\mathcal{K}$ is hyperbolic for the flow $\varphi^{t}$, if the generator $X$ of the flow does not vanish on $\mathcal{K}$ and each tangent space $T_{x} \mathcal{M}, x \in \mathcal{K}$, admits a flow/stable/unstable decomposition

$$
T_{x} \mathcal{M}=E_{0}(x) \oplus E_{s}(x) \oplus E_{u}(x), \quad x \in \mathcal{K},
$$

such that

- $E_{0}(x)=\mathbb{R} X(x)$;

- $E_{s}(x), E_{u}(x)$ depend continuously on the point $x$;

- $d \varphi^{t}\left(E_{s}(x)\right)=E_{s}\left(\varphi^{t}(x)\right)$ and $d \varphi^{t}\left(E_{u}(x)\right)=E_{u}\left(\varphi^{t}(x)\right)$ for all $x \in \mathcal{K}, t \in \mathbb{R}$;

- for any choice of a continuous norm $|\bullet|$ on the fibers of $T \mathcal{M}$, there exist constants $C, \theta>0$ such that for all $x \in \mathcal{K}$,

$$
\left|d \varphi^{t}(x) v\right| \leq C e^{-\theta|t|}|v| \quad \text { when } \begin{cases}t \geq 0, & v \in E_{s}(x) \\ t \leq 0, & v \in E_{u}(x) .\end{cases}
$$

We say $\varphi^{t}$ is an Anosov flow if the whole $\mathcal{M}$ is hyperbolic.

A fixed point $x \in \mathcal{M}, X(x)=0$, is called hyperbolic if the differential $\nabla X(x)$ has no eigenvalues on the imaginary axis. Hyperbolic fixed points are nondegenerate and thus isolated. 
We also define the nonwandering set:

Definition 2.2 (Smale [Sm67, p. 796]). We call $x \in \mathcal{M}$ a nonwandering point if for every neighborhood $V$ of $x$ and every $T>0$ there exists $t \in \mathbb{R}$ such that $|t| \geq T$ and $\varphi^{t}(V) \cap V \neq \emptyset$. The set of all nonwandering points is called the nonwandering set.

The nonwandering set is closed and $\varphi^{t}$-invariant; see [KaHa97, Proposition 3.3.4]. Note that each closed orbit of $\varphi^{t}$ lies in the nonwandering set.

We now give the definition of Axiom A flows:

Definition 2.3 (Smale [Sm67, $\S I I .5,(5.1)]$ ). The flow $\varphi^{t}$ is Axiom $A$ if

(1) the nonwandering set is the disjoint union of the set $\mathcal{F}$ of fixed points and the closure $\mathcal{K}$ of the union of all closed orbits;

(2) all fixed points of $\varphi^{t}$ are hyperbolic;

(3) the set $\mathcal{K}$ is hyperbolic for the flow $\varphi^{t}$.

We also define locally maximal sets and basic hyperbolic sets:

Definition 2.4. We say that a compact $\varphi^{t}$-invariant set $K \subset \mathcal{M}$ is locally maximal for the flow $\varphi^{t}$ if there exists a neighborhood $V$ of $K$ such that

$$
K=\bigcap_{t \in \mathbb{R}} \varphi^{t}(V) .
$$

Definition 2.5 (Parry and Pollicott $\left[\right.$ PaPo83, Chapter 9]). A compact $\varphi^{t}$-invariant set $K \subset \mathcal{M}$ is called a basic hyperbolic set for $\varphi^{t}$ if

(1) $K$ is locally maximal for $\varphi^{t}$;

(2) $K$ is hyperbolic for $\varphi^{t}$;

(3) the flow $\left.\varphi^{t}\right|_{K}$ topologically transitive (i.e., contains a dense orbit); and

(4) $K$ is the closure of the union of all closed orbits of $\left.\varphi^{t}\right|_{K}$.

The nonwandering set of an Axiom A flow has the following spectral decomposition:

Proposition 2.6 (Smale [Sm67, §II.5, Theorem 5.2]). Assume that $\varphi^{t}$ is an Axiom $A$ flow, and let $\mathcal{K}$ be given by Definition 2.3 . Then we can write $\mathcal{K}$ as a finite disjoint union

$$
\mathcal{K}=K_{1} \sqcup \cdots \sqcup K_{N},
$$

where each $K_{j}$ is a basic hyperbolic set.

See also KaHa97, Exercise 18.3.7] for a proof of the spectral decomposition, and [Bo75, Lemma 3.9] for the local maximality of the nonwandering set.

We finally give a few examples:

Example 2.7. Let $\mathcal{M}=\mathbb{R}_{x, y}^{2} / 2 \pi \mathbb{Z}^{2}$ be the torus, and let $\varphi_{t}=\exp (t X)$, where $X=(\sin x) \partial_{x}+\partial_{y}$. The nonwandering set $\mathcal{K}$ has two connected components, each being a single closed orbit:

$$
\mathcal{K}=\{x=0\} \sqcup\{x=\pi\} .
$$

The spectral decomposition is given by equation (2.2). Note however that the entire $\mathcal{M}$ is not hyperbolic for $\varphi^{t}$.

\footnotetext{
${ }^{\dagger}$ Strictly speaking, a single closed orbit is not considered a basic set, but we ignore this minor detail here.
} 
Example 2.8. Let $(M, g)$ be a (possibly noncompact) complete Riemannian manifold of negative sectional curvature which is asymptotically hyperbolic. Put $\mathcal{M}:=$ $S M$, the sphere bundle of $M$, and let $\varphi^{t}: \mathcal{M} \rightarrow \mathcal{M}$ be the geodesic flow. Let $\mathcal{K}$ be the union of all geodesics which are trapped, that is their closures in $S M$ are compact. Then $\mathcal{K}$ is a locally maximal hyperbolic set for $\varphi^{t}$. (The noncompactness of $\mathcal{M}$ is not an issue since $\mathcal{K}$ is compact and the behavior of the flow outside of a neighborhood of $\mathcal{K}$ is irrelevant.) See DyGu16, §6.3] for details and more general examples.

\section{Proof of the theorem}

We first show meromorphic continuation of the Ruelle zeta function $\zeta_{K}$ for a locally maximal hyperbolic set:

Proposition 3.1. Let $K \subset \mathcal{M}$ be a locally maximal hyperbolic set for $\varphi^{t}$. Define the Ruelle zeta function $\zeta_{K}$ by equation (1.1), where we only take the closed trajectories of $\varphi^{t}$ which lie in $K$. Then $\zeta_{K}$ continues to a meromorphic function on $\mathbb{C}$.

The proof of Proposition 3.1 relies on DyGu16. To reduce to the case considered in DyGu16, we use

Lemma 3.2. Let $K \subset \mathcal{M}$ be a locally maximal hyperbolic set for $\varphi^{t}$. Then there exists a neighborhood $\mathcal{U}$ of $K$ in $\mathcal{M}$ with $C^{\infty}$ boundary and a $C^{\infty}$ vector field $X_{0}$ on $\overline{\mathcal{U}}$ such that

(1) the boundary $\partial \mathcal{U}$ is strictly convex with respect to $X_{0}$ in the sense that

$$
\rho(x)=0, \quad X_{0} \rho(x)=0 \quad \Longrightarrow \quad X_{0}^{2} \rho(x)<0,
$$

where $\rho \geq 0$ is any boundary defining function on $\mathcal{U}$;

(2) $X_{0}=X$ in a neighborhood of $K$;

(3) $K=\bigcap_{t \in \mathbb{R}} \varphi_{0}^{t}(\mathcal{U})$, where $\varphi_{0}^{t}:=\exp \left(t X_{0}\right)$ is the flow generated by $X_{0}$.

Proof. The proof follows Guillarmou, Mazzucchelli, and Tzou [GMT17, Lemma 2.3]. We first use a result of Conley and Easton [CoEa71, Theorem 1.5]. Since $K$ is locally maximal for $\varphi^{t}$, there exists an open set $V \subset \mathcal{M}$ containing $K$ such that $K=\bigcap_{t \in \mathbb{R}} \varphi^{t}(\bar{V})$. If $x \in \partial V$, then in particular $x \notin K$, thus there exists $t \in \mathbb{R}$ such that $\varphi^{t}(x) \notin \bar{V}$. Therefore, $V$ is an isolating neighborhood for $\varphi^{t}$ in the sense of [CoEa71, Definition 1.1], and $K$ is an isolated invariant set in the sense of [CoEa71, Definition 1.2]. Therefore, by [CoEa71, Theorem 1.5] there exists an isolating block, which is an open subset $\mathcal{U} \subset \mathcal{M}$ with $K \subset \mathcal{U}$ with the following properties:

- $\mathcal{U}$ has compact closure and $C^{\infty}$ boundary, that is, $\mathcal{U}=\{x \in \mathcal{M} \mid \rho(x)>0\}$ for some function $\rho \in C^{\infty}(\mathcal{M} ; \mathbb{R})$ such that $d \rho \neq 0$ on $\partial \mathcal{U}=\{x \in \mathcal{M} \mid$ $\rho(x)=0\}$;

- the set $\partial_{0} \mathcal{U}:=\left\{x \in \partial \mathcal{U} \mid X_{0} \rho(x)=0\right\}$ is a codimension 1 smooth submanifold of $\partial \mathcal{U}$;

- for each $x \in \partial_{0} \mathcal{U}$, there exists $\varepsilon>0$ such that

$$
\varphi^{t}(x) \notin \overline{\mathcal{U}} \text { for all } t \in(-\varepsilon, \varepsilon) \backslash\{0\} .
$$

Now the vector field $X_{0}$ is obtained by modifying $X$ slightly near $\partial_{0} \mathcal{U}$ so that we still have $K=\bigcap_{t \in \mathbb{R}} \varphi_{0}^{t}(\overline{\mathcal{U}})$, and the topological (local) convexity condition (3.2) is replaced by the quadratic differential convexity condition (3.1). We refer to the proof of GMT17, Lemma 2.3] for details. 
We now give

Proof of Proposition 3.1. By part (3) of Lemma 3.2, every closed trajectory of $\varphi_{0}^{t}$ in $\overline{\mathcal{U}}$ lies in $K$ and thus is a closed trajectory of $\varphi^{t}$. Therefore, the Ruelle zeta function $\zeta_{K}$ is equal to the Ruelle zeta function of $\varphi_{0}^{t}$ on $\overline{\mathcal{U}}$. Now $\left(\mathcal{U}, \varphi_{0}^{t}\right)$ is an open hyperbolic system in the sense of DyGu16, Assumptions (A1)-(A4)]. Therefore DyGu16, Theorem 3] applies (with potential set to 0) and gives a meromorphic continuation of $\zeta_{K}$ to $\mathbb{C}$.

The main theorem now follows using the spectral decomposition theorem, Proposition 2.6. Indeed, if $\mathcal{K}=K_{1} \sqcup \cdots \sqcup K_{N}$ is the spectral decomposition of $\varphi^{t}$, then each closed trajectory of $\varphi^{t}$ is contained in one of the sets $K_{j}$. Therefore, the Ruelle zeta function (1.1) factorizes as

$$
\zeta(\lambda)=\zeta_{K_{1}}(\lambda) \cdots \zeta_{K_{N}}(\lambda)
$$

Since each $\zeta_{K_{j}}$ admits a meromorphic continuation to $\mathbb{C}$ by Proposition 3.1, the function $\zeta$ admits a meromorphic continuation to $\mathbb{C}$ as well.

\section{REFERENCES}

[Ba17] Viviane Baladi, Dynamical zeta functions and dynamical determinants for hyperbolic maps: a functional approach, to appear in Ergebnisse der Mathematik und ihrer Grenzgebiete, Springer.

[BaTs07] Viviane Baladi and Masato Tsujii, Anisotropic Hölder and Sobolev spaces for hyperbolic diffeomorphisms (English, with English and French summaries), Ann. Inst. Fourier (Grenoble) 57 (2007), no. 1, 127-154. MR2313087

[BKL02] Michael Blank, Gerhard Keller, and Carlangelo Liverani, Ruelle-Perron-Frobenius spectrum for Anosov maps, Nonlinearity 15 (2002), no. 6, 1905-1973, DOI 10.1088/09517715/15/6/309. MR.1938476

[Bo75] Rufus Bowen, Equilibrium states and the ergodic theory of Anosov diffeomorphisms, Lecture Notes in Mathematics, Vol. 470, Springer-Verlag, Berlin-New York, 1975. MR.0442989

[BuLi07] Oliver Butterley and Carlangelo Liverani, Smooth Anosov flows: correlation spectra and stability, J. Mod. Dyn. 1 (2007), no. 2, 301-322, DOI 10.3934/jmd.2007.1.301. MR2285731

[CoEa71] C. Conley and R. Easton, Isolated invariant sets and isolating blocks, Trans. Amer. Math. Soc. 158 (1971), 35-61, DOI 10.2307/1995770. MR0279830

[DyGu16] Semyon Dyatlov and Colin Guillarmou, Pollicott-Ruelle resonances for open systems, Ann. Henri Poincaré 17 (2016), no. 11, 3089-3146, DOI 10.1007/s00023-016-0491-8. MR.3556517

[DyZw16] Semyon Dyatlov and Maciej Zworski, Dynamical zeta functions for Anosov flows via microlocal analysis (English, with English and French summaries), Ann. Sci. Éc. Norm. Supér. (4) 49 (2016), no. 3, 543-577, DOI 10.24033/asens.2290. MR3503826

[FRS08] Frédéric Faure, Nicolas Roy, and Johannes Sjöstrand, Semi-classical approach for Anosov diffeomorphisms and Ruelle resonances, Open Math. J. 1 (2008), 35-81, DOI 10.2174/1874117700801010035. MR2461513

[FaSj11] Frédéric Faure and Johannes Sjöstrand, Upper bound on the density of Ruelle resonances for Anosov flows (English, with English and French summaries), Comm. Math. Phys. 308 (2011), no. 2, 325-364, DOI 10.1007/s00220-011-1349-z. MR2851145

[FaTs17] Frédéric Faure and Masato Tsujii, The semiclassical zeta function for geodesic flows on negatively curved manifolds, Invent. Math. 208 (2017), no. 3, 851-998, DOI 10.1007/s00222-016-0701-5. MR3648976

[GLP13] P. Giulietti, C. Liverani, and M. Pollicott, Anosov flows and dynamical zeta functions, Ann. of Math. (2) 178 (2013), no. 2, 687-773, DOI 10.4007/annals.2013.178.2.6. MR.3071508 
[GoLi08] Sébastien Gouëzel and Carlangelo Liverani, Compact locally maximal hyperbolic sets for smooth maps: fine statistical properties, J. Differential Geom. 79 (2008), no. 3, 433-477. MR2433929

[GMT17] Colin Guillarmou, Marco Mazzucchelli, and Leo Tzou, Boundary and lens rigidity for non-convex manifolds, preprint, arXiv:1711.10059.

[KaHa97] Anatole Katok and Boris Hasselblatt, Introduction to the modern theory of dynamical systems, Encyclopedia of Mathematics and its Applications, vol. 54, Cambridge University Press, Cambridge, 1995. With a supplementary chapter by Katok and Leonardo Mendoza. MR 1326374

[Ki99] A. Yu. Kitaev, Fredholm determinants for hyperbolic diffeomorphisms of finite smoothness, Nonlinearity 12 (1999), no. 1, 141-179, DOI 10.1088/0951-7715/12/1/008. MR.1668543

[Li04] Carlangelo Liverani, On contact Anosov flows, Ann. of Math. (2) 159 (2004), no. 3, 1275-1312, DOI 10.4007/annals.2004.159.1275. MR2113022

[PaPo83] William Parry and Mark Pollicott, An analogue of the prime number theorem for closed orbits of Axiom A flows, Ann. of Math. (2) 118 (1983), no. 3, 573-591, DOI 10.2307/2006982. MR727704

[Sm67] S. Smale, Differentiable dynamical systems, Bull. Amer. Math. Soc. 73 (1967), 747-817, DOI 10.1090/S0002-9904-1967-11798-1. MR.0228014

Department of Mathematics, University of California, Berkeley, California

Email address: dyatlov@math.berkeley.edu

Laboratoire de Mathématiques d'Orsay, Faculté des Sciences d'Orsay Université Paris-Sud, F-91405 Orsay Cedex, France

Email address: cguillar@math.cnrs.fr 